\title{
Epidermolysis Bullosa with Congenital Pyloric Atresia: Novel Mutations in the $\beta 4$ Integrin Gene (ITGB4) and Genotype/Phenotype Correlations
}

\author{
AOI NAKANO, LEENA PULKKINEN, DEDEE MURRELL, JOYCE RICO, ANNE W. LUCKY, \\ MARIA GARZON, CATHY A. STEVENS, STEPHEN ROBERTSON, ELLEN PFENDNER, \\ AND JOUNI UITTO
}

Departments of Dermatology and Cutaneous Biology, and Biochemistry and Molecular Pharmacology, Jefferson Medical College, Jefferson Institute of Molecular Medicine, Thomas Jefferson University, Philadelphia, Pennsylvania 19107, U.S.A. [A.N., L.P., E.P., J.U.]; Department of Dermatology, St. George Hospital, University of South Wales, Sydney, New South Wales, 2217, Australia [D.M.]; Department of Dermatology, New York University Medical Center, New York, New York 10016, U.S.A. [J.R.]; Children's Hospital Medical Center, Cincinnati, Ohio 45229, U.S.A. [A.W.L.]; Departments of Dermatology and Pediatrics, Columbia University, New York, New York 10032, U.S.A. [M.G.]; Department of Pediatrics, T.C. Thompson Children's Hospital, Chattanooga, Tennessee 37403, U.S.A. [C.A.S.]; Victorian Clinical Genetics Service, Royal Children's Hospital, Melbourne, Victoria, 3052, Australia [S.R.]

\begin{abstract}
Epidermolysis bullosa with pyloric atresia (EB-PA: OMIM 226730), also known as Carmi syndrome, is a rare autosomal recessive genodermatosis that manifests with neonatal mucocutaneous fragility associated with congenital pyloric atresia. The disease is frequently lethal within the first year, but nonlethal cases have been reported. Mutations in the genes encoding subunit polypeptides of the $\alpha 6 \beta 4$ integrin (ITGA6 and ITGB4) have been demonstrated in EB-PA patients. To extend the repertoire of mutations and to identify genotype-phenotype correlations, we examined seven new EB-PA families, four with lethal and three with nonlethal disease variants. DNA from patients was screened for mutations using heteroduplex analysis followed by nucleotide sequencing of PCR products spanning all $\beta 4$ integrincoding sequences. Mutation analysis disclosed 12 distinct mutations, 11 of them novel. Four mutations predicted a premature termination codon as a result of nonsense mutations or small out-of-frame insertions or deletions, whereas seven were missense mutations. This brings the total number of distinct ITGB4 mutations to 33 . The mutation database indicates that premature
\end{abstract}

\section{ABSTRACT}

termination codons are associated predominantly with the lethal EB-PA variants, whereas missense mutations are more prevalent in nonlethal forms. However, the consequences of the missense mutations are position dependent, and substitutions of highly conserved amino acids may have lethal consequences. In general, indirect immunofluorescence studies of affected skin revealed negative staining for $\beta 4$ integrin in lethal cases and positive, but attenuated, staining in nonlethal cases and correlated with clinical phenotype. The data on specific mutations in EB-PA patients allows prenatal testing and preimplantation genetic diagnosis in families at risk. (Pediatr Res 49: 618-626, 2001)
EB-PA, epidermolysis bullosa with pyloric atresia
BMZ, basement membrane zone
CSGE, conformation-sensitive gel electrophoresis
PTC, premature termination codon
TEM, transmission electron microscopy
ITGB4, $\beta 4$ integrin gene

Epidermolysis bullosa (EB) is a group of genodermatoses manifesting with blistering and erosions of the skin and mu-

Received April 7, 2000; accepted July 18, 2000.

Correspondence: Dr. Jouni Uitto, Department of Dermatology and Cutaneous Biology, Jefferson Medical College, 233 S. 10th Street, Suite 450 BLSB, Philadelphia, PA 19107, U.S.A.; e-mail: Jouni.Uitto@mail.tju.edu

Supported by the United States Public Health Service, National Institutes of Health Grant PO1 AR 38923. cous membranes as a result of mechanical trauma $(1,2)$. The clinical severity of EB is highly variable. In the mildest cases, blistering occurs primarily on the hands and feet with relatively little morbidity, whereas, in the most severe cases, the disease can be lethal perinatally or during the early postnatal period. Traditionally, EB has been divided into three broad categories based on clinical findings and ultrastructural demonstrations of 
the level of tissue separation within the cutaneous BMZ (3). In the simplex forms of EB (EBS), tissue separation is intraepidermal, and mutations in the basal cell keratin genes, KRT5 and KRT14, underlie the classic forms of EBS inherited in an autosomal dominant fashion. In the junctional forms of EB (JEB), blistering occurs within the lamina lucida of the BMZ, and, in the classic Herlitz JEB, mutations in any of the three genes (LAMA3a, LAMB3, and LAMC2) encoding the subunit polypeptides of laminin 5 underlie this form of EB. In the dystrophic forms of EB (DEB), tissue separation occurs below the lamina densa on the dermal side of the BMZ, and mutations in the gene COL7A1 encoding type VII collagen, the major if not the exclusive component of the anchoring fibrils, have been disclosed in this form of EB (4). Adding to the heterogeneity of EB is the fact that there are, in addition to skin involvement, various extracutaneous manifestations associated with distinct subtypes of EB. These include corneal erosions, enamel pitting, nail dystrophy, scarring alopecia, muscle weakness, and detachment of the respiratory epithelium, as well as involvement of the gastrointestinal and vesico-urinary tract (1).

Recently, a new category, the hemidesmosomal variants of EB, has been proposed, and clinically there are three distinct subtypes within this category (5). First, generalized atrophic benign EB (GABEB) is characterized by life-long blistering of the skin associated with hair and tooth abnormalities. Traditionally, this subtype has been classified as a nonlethal variant of junctional $\mathrm{EB}(6,7)$. It is now known that the majority of patients with GABEB harbor mutations in the type XVII collagen/180-kD bullous pemphigoid antigen gene (COL17A1/BPAG2) (5). Secondly, a distinct variant of EB is characterized by relatively mild blistering of the skin noted at birth, associated with late-onset muscular dystrophy (EB-MD) (8). The onset of the muscle involvement in EB-MD is highly variable, so that in some cases the muscle weakness is present within a few years of age, whereas in others the muscle weakness is not noted until the third or fourth decade of life. Nevertheless, this combination of skin and muscle involvement is now known to result from mutations in the gene encoding plectin, a large adhesion molecule with wide tissue distribution including expression in the hemidesmosomes of the skin as well as in the sarcolemma and Z-lines of the muscle (8). Finally, a distinct variant of EB presents with a combination of perinatal or neonatal blistering of the skin associated with congenital pyloric atresia (EB-PA) $(1,5)$. Diagnosis of pyloric atresia requires immediate surgical intervention, but the severity of the cutaneous and extracutaneous manifestations can be variable. In some cases, the blistering is extremely severe and can result in early demise of the affected individuals, whereas, in others, a blistering tendency has not been noted until several months or even a few years after the birth (9-13). Also, in some cases, the blistering tendency is initially severe but becomes progressively milder and later in life requires considerable trauma for the clinical features to manifest.

Initial clues to the candidate gene/protein systems in EB-PA were obtained from immunofluorescence analysis of skin. Specifically, markedly reduced or absent staining for $\alpha 6$ and/or $\beta 4$ integrin has been demonstrated in the skin of these patients, suggesting that the corresponding genes, ITGA6 and ITGB4, may harbor the mutations underlying this subset of EB (1416). Subsequently, a number of mutations in these two genes have been demonstrated, the majority of the mutations being found in the $\beta 4$ integrin gene $(10-13,17-20)$, whereas only three cases with mutations in the $\alpha 6$ integrin gene have been reported thus far $(9,21,22)$. Two of the $\alpha 6$ integrin cases were lethal in the early postnatal period, and in one case an affected fetus with abnormalities in the skin and intestine was detected prenatally by ultrasound examination, and the pregnancy was terminated. Subsequent immunochemical analysis of the latter case revealed altered expression of $\alpha 6 \beta 4$ integrin (22). Therefore, neither the $\alpha 6$ nor the $\beta 4$ integrin databases provide definitive explanations for the clinical variability, i.e. lethal versus nonlethal phenotype.

In this study, we have examined seven new families, each with an individual affected with EB-PA, for mutations in the genes encoding the $\alpha 6 \beta 4$ integrin subunits. We have disclosed a total of 11 novel mutations in the ITGB4 gene, and examination of the entire $\beta 4$ integrin mutation database suggests intriguing genotype/phenotype correlations.

\section{MATERIALS AND METHODS}

Clinical material and verification of the diagnosis. Seven families $(A-G)$ with the clinical diagnosis of EB-PA were referred from an international patient base to the DebRA Molecular Diagnostics Laboratory at the Department of Dermatology and Cutaneous Biology, Jefferson Medical College, Philadelphia, PA, U.S.A. All studies from this laboratory have appropriate Internal Review Board approval. In each family, the proband had history of skin blistering, first noted at birth or shortly thereafter, associated with congenital pyloric atresia. Peripheral blood samples were submitted after obtaining informed consent and DNA was isolated by phenol-chloroform extraction using a standard procedure (23). Samples were obtained from the proband and both parents, if available. Control DNA was obtained from 100 unrelated individuals with no evidence of a blistering skin disease. DNA was used as a template for PCR amplification of exons of ITGB4 and ITGA6 for mutation detection analyses (see below).

Diagnostic skin biopsy specimens were obtained from the probands and subjected to routine histopathologic examination, and five specimens were examined by TEM. Immunofluorescence staining of frozen skin sections with antibodies recognizing $\alpha 6$ (GoH3) and $\beta 4$ (3E1) integrins was performed. Antibodies recognizing other BMZ components, including type VII collagen (LH7.2 and NP185), laminin 5 (GB3), bullous pemphigoid antigen (patient sera), and plectin (HD121) were used in comparative immunofluorescence staining of some samples (Table 1).

Characterization and verification of mutations. Mutation detection strategy used in this study included PCR amplification of each exon and of the flanking intronic sequences of the ITGB4 gene in each family, followed by heteroduplex scanning of the PCR products by CSGE, as described previously (11). The primer sequences used for amplification of each of the 39 coding exons expressed in the skin (numbers 2-32, $34-41$ ) of the ITGB4 gene have been reported elsewhere (11). 
Table 1. Clinical features of patients with epidermolysis bullosa and pyloric atresia

\begin{tabular}{|c|c|c|c|c|c|c|}
\hline Family & $\begin{array}{l}\text { Proband } \\
\text { age/sex }\end{array}$ & Clinical features* & $\begin{array}{l}\text { Disease } \\
\text { variant }\end{array}$ & $\mathrm{EM} / \mathrm{IF}$ features $\dagger$ & ITGB4 mutations & Consequences \\
\hline A & $\begin{array}{l}\text { Demise at } \\
4.5 \mathrm{mo} / \mathrm{F}\end{array}$ & $\begin{array}{l}\text { Mild blistering at birth, anemia, respiratory } \\
\text { distress, gastro-esophageal reflux }\end{array}$ & Lethal & $\begin{array}{l}\text { Lamina lucida cleavage, } \\
\text { reduced HDs, } \beta 4 \text { negative, } \\
\alpha 6 \text { negative, plectin reduced, } \\
\text { BPAG reduced }\end{array}$ & 658delC/R252C & PTC/missense \\
\hline $\mathrm{B}$ & $\begin{array}{l}\text { Demise at } 2 \\
\mathrm{mo} / \mathrm{M}\end{array}$ & $\begin{array}{l}\text { Aplasia cutis congenita of all four extremities; } \\
\text { multicystic dysplasia of left kidney; } \\
\text { hydronephrosis of right kidney; esophageal, } \\
\text { tracheal, bladder, and small intestinal } \\
\text { involvement }\end{array}$ & Lethal & $\begin{array}{l}\beta 4 \text { negative, } \alpha 6 \text { negative, } \\
\text { plectin altered }\end{array}$ & D131Y/G273D & $\begin{array}{l}\text { Missense/ } \\
\text { missense }\end{array}$ \\
\hline $\mathrm{C}$ & $\begin{array}{l}\text { Alive at } 15 \\
\mathrm{mo} / \mathrm{F}\end{array}$ & Severe blistering at birth; absent at $1 \mathrm{y}$ & Nonlethal & $\begin{array}{l}\text { Hemidesmosomal cleavage, } \beta 4 \\
\text { reduced, } \alpha 6 \text { reduced, plectin } \\
\text { altered }\end{array}$ & $\mathrm{ND} / \mathrm{R} 283 \mathrm{C} \S$ & $\mathrm{ND} /$ missense \\
\hline $\mathrm{D}$ & $\begin{array}{l}\text { Demise at } 1 \\
\mathrm{mo} / \mathrm{F}\end{array}$ & Obstructed sigmoid colon & Lethal & $\begin{array}{l}\text { Lamina lucida cleavage, } \beta 4 \\
\text { negative, } \alpha 6 \text { reduced, plectin } \\
\text { altered, BPAG negative }\end{array}$ & $\begin{array}{l}\text { 1874delTCTinsC/ } \\
\text { V325D }\end{array}$ & PTC/missense \\
\hline $\mathrm{E}$ & $\begin{array}{l}\text { Alive at } 13 \\
\mathrm{yrs} / \mathrm{F}\end{array}$ & $\begin{array}{l}\text { Urologic obstruction, ocular involvement, } \\
\text { scarring on legs, hypoplastic enamel, } \\
\text { laryngeal obstruction, }\end{array}$ & Nonlethal & $\begin{array}{l}\beta 4 \text { normal, } \alpha 6 \text { normal, plectin } \\
\text { normal/altered }\end{array}$ & $\begin{array}{l}3112-3 \mathrm{C} \rightarrow \mathrm{T}, \\
\mathrm{L} 336 \mathrm{P} / \mathrm{R} 1225 \mathrm{H}\end{array}$ & $\begin{array}{l}\mathrm{Spl}(?) \ddagger \\
\text { missense/ } \\
\text { missense }\end{array}$ \\
\hline $\mathrm{F}$ & $\begin{array}{l}\text { Demise at } \\
2.5 \mathrm{mo} / \mathrm{M}\end{array}$ & Mild blistering at birth & Lethal & Intraepidermal cleavage & Q1767X/Q1767X & PTC/PTC \\
\hline G & $\begin{array}{l}\text { Alive at } 13 \\
\mathrm{mo} / \mathrm{F}\end{array}$ & Mild blistering, severe corneal erosions & Nonlethal & $\begin{array}{c}\text { Hypoplastic HDs, absence of } \\
\text { subbasal dense plate, lamina } \\
\text { lucida cleavage, } \beta 4 \text { reduced, } \\
\alpha 6 \text { reduced, plectin altered }\end{array}$ & $\begin{array}{l}\text { 4790delTC/ } \\
\text { 4790delTC }\end{array}$ & PTC/PTC \\
\hline
\end{tabular}

* All patients demonstrated blistering and erosions at birth, as well as congenital pyloric atresia. Additional clinical features are listed in this column.

$\dagger E M$, electron microscopy; $I F$, immunofluorescence; $H D$, hemidesmosome; BPAG2, bullous pemphigoid antigen; for IF see Figs. 1 and 2

$\ddagger$ ND, not detected; Spl(?), a putative splice-site mutation

$\S$ Sequencing of the $\alpha 6$ integrin gene (ITGA6) did not reveal pathogenic mutations.

The PCR reactions were performed in a total volume of 50 $\mu \mathrm{L}$ containing $1 \times$ PCR buffer, $200 \mu \mathrm{M}$ of each nucleotide, and either $1.25 \mathrm{U}$ of Taq polymerase in the presence of $1 \times$ Q-buffer (Qiagen, Valencia, CA, U.S.A.) or $1.25 \mathrm{U}$ of Amplitaq polymerase (Perkin Elmer, Foster City, CA, U.S.A.) in the presence of 4\% DMSO, 12.8 pmol of each primer, and $200 \mathrm{ng}$ of genomic DNA. The amplification conditions were as follows: $5 \mathrm{~min}$ at $94^{\circ} \mathrm{C}$ for one cycle, followed by 38 cycles of $45 \mathrm{~s}$ at $94^{\circ} \mathrm{C}, 45 \mathrm{~s}$ at the annealing temperature (11), and $45 \mathrm{~s}$ at $72^{\circ} \mathrm{C}$. The PCR products were analyzed on $2 \%$ agarose gel and subjected to heteroduplex analysis by CSGE, as described elsewhere $(24,25)$. All PCR products demonstrating heteroduplexes, suggesting the presence of a sequence variant, were directly sequenced by an automated DNA sequencer (ABI 377, Perkin Elmer).

In each family, the verification of the mutation was performed by digesting the PCR products representing the proband and his or her immediate family members by restriction endonucleases (Pst I and Nar I for family A; Ava II and $\mathrm{Hga}$ I for family B; $A c i$ I for family C; Apa I and $H p h$ I for family D; HindIII, $B f a$ I and $B s a H$ I for family E; $A p a$ I for family F; and $B s m A$ I for family $\mathrm{G}$ ). For detection of the mutation 4790delTC in family $\mathrm{G}$, specific primers were designed so that the $\mathrm{C}$ nucleotide at position 4787 was replaced by a $\mathrm{G}$ (underlined) in the forward primer: L, 5'-TTCTCTGCCCTGGGGC CCACATGTC-3', R, 5'-TTAGCGCTCAAGGGACTTGG-3'.

\section{RESULTS AND DISCUSSION}

Clinical and genetic features of patients with EB-PA. A total of seven families, each with an affected child with EB-PA, were subjected to study (Table 1). In each case, except family F (Table 1), there was no family history of a blistering disease, and the parents in each case were clinically unaffected. Family $F$ lived in an isolated aboriginal community where similar cases were reported, one of which was in a distant family member (26). Typically, the affected newborn was diagnosed as having pyloric atresia that required surgical intervention, and, in most cases, skin blistering was noted at birth or shortly thereafter (Fig. 1A). In one case, family B (Table 1), the diagnosis of aplasia cutis congenita was also made at the time of birth. The absence of skin was particularly extensive, covering approximately $30 \%$ of the entire skin surface on all extremities. Among the seven probands, four of them (families A, B, D, and F) died within the first year of life from complications of EB-PA, and these individuals were classified as having the lethal variant of EB-PA. In contrast, in three of the patients (families $\mathrm{C}, \mathrm{E}$, and $\mathrm{G}$ ) the blistering tendency improved with time. For example, a 13-y-old patient (family E), a ballet dancer, who had extensive blistering involving skin and the mucous membranes at birth, showed relatively mild skin fragility at the time of this study, and blisters develop on her toes only when she is dancing en pointe. She continues, however, to note increased skin fragility on her upper arms and trunk, to the extent that adhesive tape and bandages will induce blisters and erosions. Thus, the patients surviving beyond $1 \mathrm{y}$ of age have been classified as nonlethal variants of EB-PA.

Several of the individuals also exhibited extracutaneous manifestations in addition to pyloric atresia $(27,28$; Kambham et al., unpublished data). For example, vesico-urinary tract involvement was noted in patient $\mathrm{E}$ at approximately $3 \mathrm{y}$ of age 

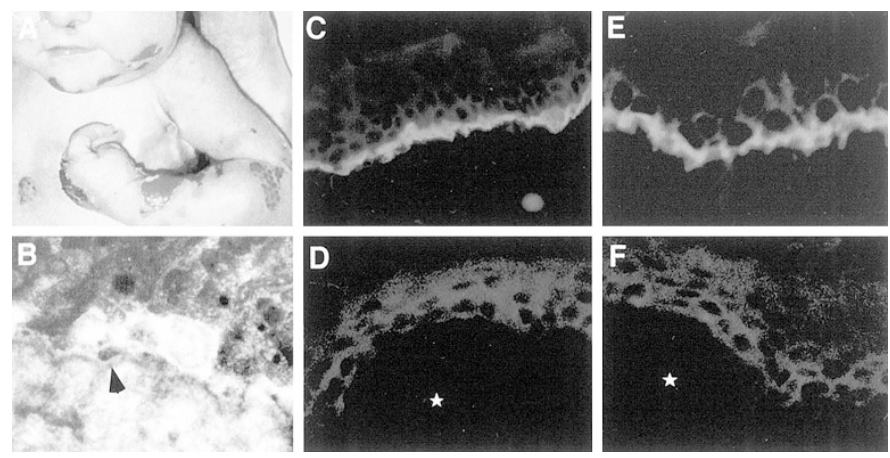

Figure 1. Clinical features, electron microscopy, and immunofluorescence staining of the skin of proband in family A. $(A)$ The affected infant at $10 \mathrm{~d}$ of age shows multiple erosions. $(B)$ Electron micrograph of the proband's skin shows separation at the level of lamina lucida and hypoplastic hemidesmosomes, sometimes on the floor of the blister (arrow). $(C-F)$ Immunofluorescence mapping of a skin biopsy taken from freshly traumatized skin from the proband using normal human skin as a control. Both of the $3 \mathrm{E} 1$ and the $\mathrm{GoH} 3$ staining recognizing $\beta 4$ and $\alpha 6$ integrin polypeptides, respectively, are negative in the proband's skin $(D, F)$ compared with normal skin $(C, E)$. Blister cavity of patient's skin is indicated by asterisks.

as a result of increased blood pressure and included ureteral obstruction that resulted in hydronephrosis and chronic renal failure. The patient is now a candidate for renal transplant and the progress of renal failure may require dialysis in the near future. This patient also had evidence of laryngeal obstruction and ocular abnormalities. Patient B also had kidney involvement including multicystic dysplasia and hydronephrosis as well as involvement of tracheal, esophageal, and small intestinal tissues. Patient $\mathrm{G}$ had relatively mild skin involvement but had severe corneal erosions with considerable morbidity (Table 1).

Confirmation of the diagnosis by immunofluorescence and electron microscopy. In all cases, skin biopsy specimens were subjected to histopathology as well as diagnostic immunofluorescence staining and/or TEM. Hematoxylin-eosin stain showed dermal-epidermal blistering. In five cases (families A-D and G), immunofluorescence with antibodies recognizing the $\alpha 6$ and $\beta 4$ integrins was entirely negative or weakly positive (Fig. 1, $C-F$, and Fig. 2). In family E, staining for $\beta 4$ integrin (Fig. 2) and $\alpha 6$ integrin (not shown) was normal. Basement membrane-specific staining with HD121 for plectin was clearly reduced in patients $\mathrm{B}, \mathrm{C}, \mathrm{D}$, and $\mathrm{G}$, whereas in patient $\mathrm{E}$ it was close to normal (Fig. 2). No immunofluorescence data were available from family $\mathrm{F}$.

TEM revealed tissue separation at the dermal-epidermal junction in four cases (families A, C, D, and G), the cleavage being above the lamina densa at the level of the lamina lucida or the hemidesmosomes (Fig. 1B). In family F, the separation was apparently intracellular, cytoplasmic material being noted in association with the basal lamina on the floor of the blister. Thus, all seven patients examined in this study had characteristic clinical features of EB-PA, and this diagnosis was confirmed by negative or attenuated immunofluorescence of the $\alpha 6 \beta 4$ integrin or by ultrastructural examination in six cases, the exception being family $\mathrm{E}$, in which the immunofluorescence was clearly positive for $\alpha 6 \beta 4$ integrin.

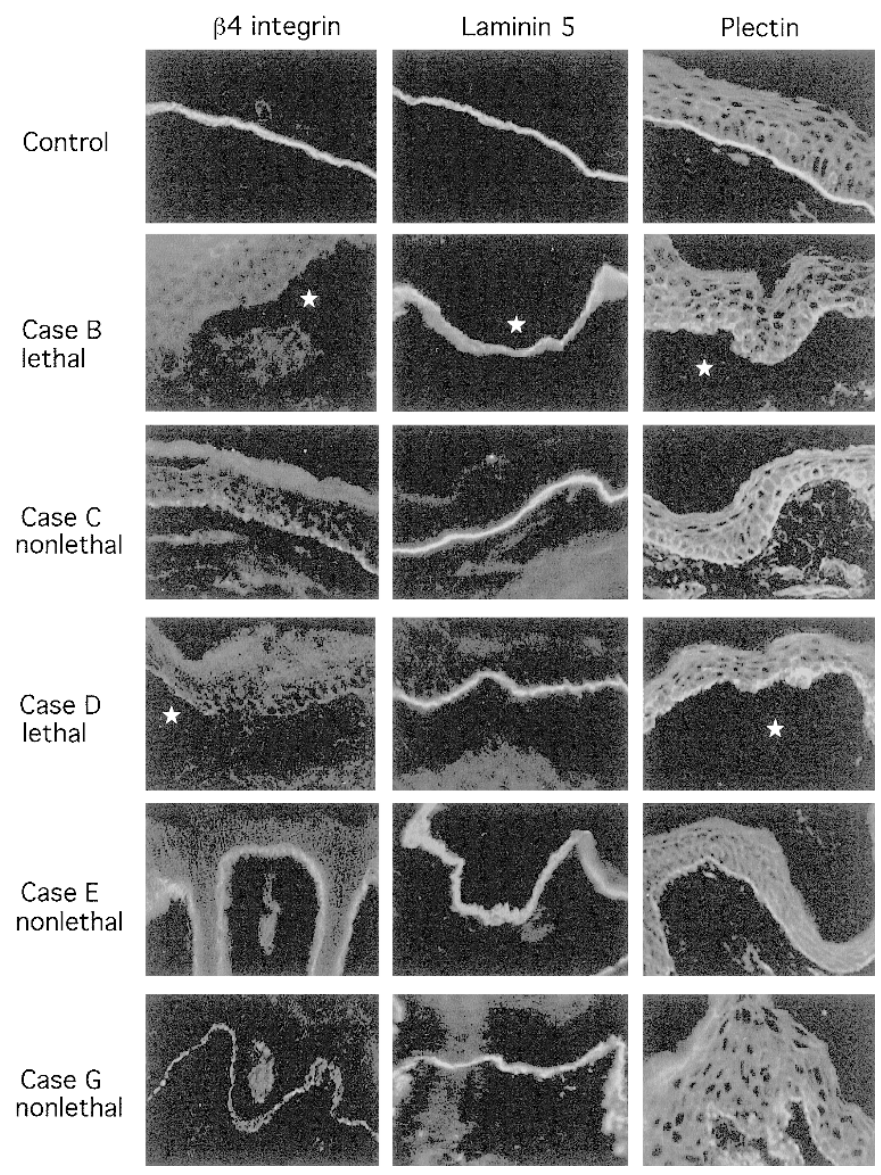

Figure 2. Immunofluorescence staining of skin sections obtained from EB-PA patients and a healthy control subject using MAb 3E1 (Chemicon International, Temecula, CA, U.S.A.), GB3 (Harlan Bioproducts for Science, Indianapolis, IN, U.S.A.), and HD121 (a gift from Dr. K. Owaribe) recognizing $\beta 4$ integrin, laminin 5 , and plectin, respectively. The linear staining was observed with all antibodies on the basement membrane of the control skin (upper panel). In addition, HD121 for plectin staining was also noted throughout the epidermis.

\section{Identification of novel mutations in the $\beta 4$ integrin gene.} To identify potential mutations in the $\alpha 6 \beta 4$ integrin genes (ITGA6 and ITGB4), a mutation detection strategy consisting of heteroduplex scanning of these genes, followed by automated nucleotide sequencing, has been established (11). First, DNA from the probands and their immediate family members, including their parents and unaffected siblings, if available, was subjected to PCR amplification of exonic sequences in ITGB4. These PCR products were then examined by CSGE; an example of the methodology is presented in Figure 3. In case of a heteroduplex, the corresponding PCR product was subjected to automated nucleotide sequencing. All sequence variants were confirmed by restriction enzyme digestion if a restriction site was altered, or by direct sequencing in other members of the family (Fig. 3).

Among the seven probands studied, 12 distinct ITGB4 mutations were found in 13 alleles of these individuals, 11 of the mutations being previously unpublished (Table 1). Among these mutations, six were missense mutations, whereas four were nonsense mutations or small out-of-frame insertions or deletions resulting in PTC downstream from the site of the 
Maternal Mutation

A
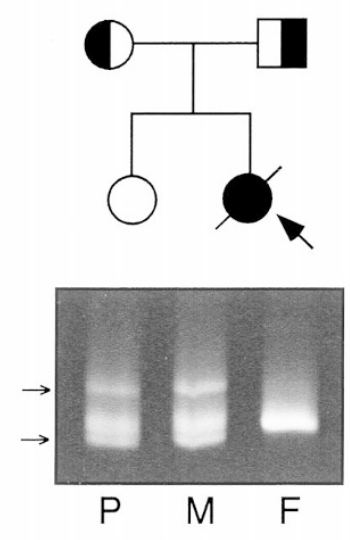

CSGE of exon 7

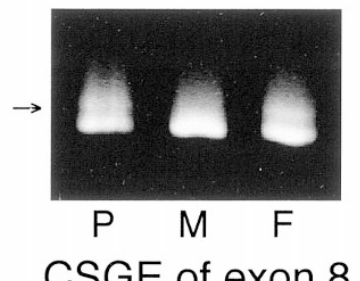

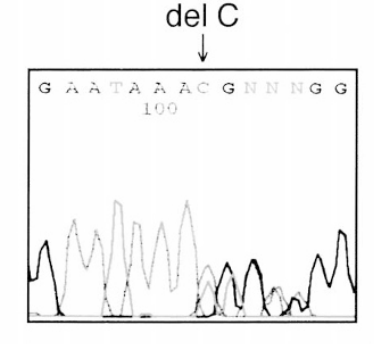

Heterozygous 658delC

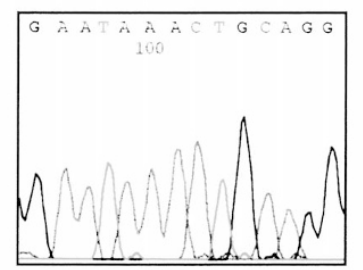

Normal sequence

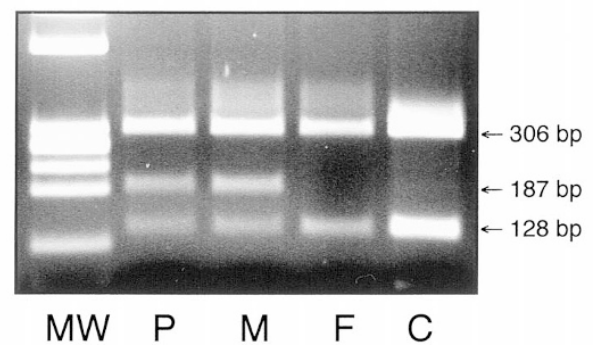

Pst I digestion of exon 7
Paternal Mutation
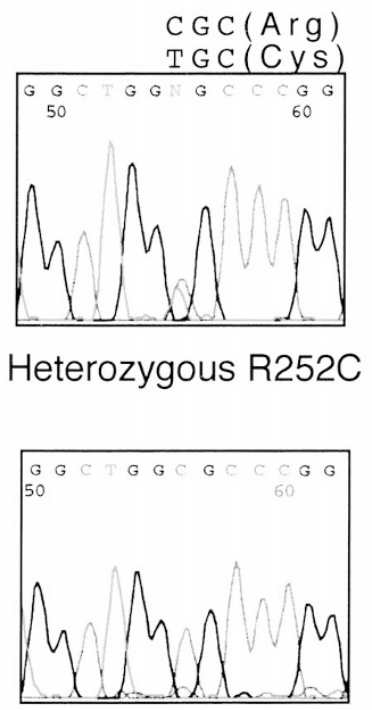

Normal sequence

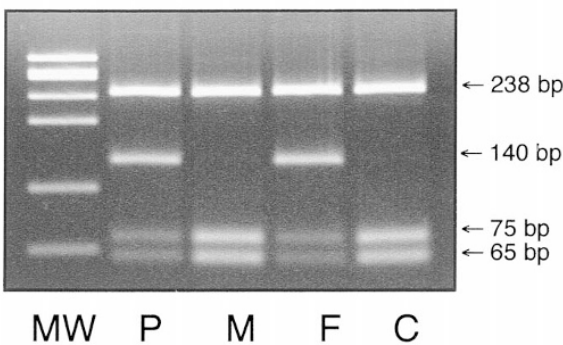

Nar I digestion of exon 8

Figure 3. Mutation analysis in family A. (A) Heteroduplex analysis of the PCR-amplified DNA spanning exon 7 and flanking intronic sequences of ITGB4 demonstrated heteroduplex bands in the mother's (lane $M$ ) and proband's (lane P) DNA (arrows), whereas the father's (lane F) DNA showed a homoduplex band only. Conversely, the father's and proband's DNA spanning exon 8 and flanking intronic sequences demonstrated heteroduplex bands (arrow), whereas the mother's DNA showed a homoduplex band only. $(B)$ Direct nucleotide sequencing of the proband's PCR product spanning exon 7 revealed the heterozygous deletion of $\mathrm{C}$ at nucleotide position 658 (658delC), as shown in the upper panel, whereas the normal sequence is shown in the middle panel. Verification of the maternal mutation by digestion with Pst I is shown in the lower panel. The mutation abolishes the restriction enzyme site for Pst I, which cuts the 494-bp PCR product in case of normal allele to 306-bp, 128-bp, and 60-bp bands, and in case of the mutant allele to 306-bp and 187-bp bands (the size difference reflects the 1-bp deletion). (C) Direct nucleotide sequencing of the proband's PCR product spanning exon 8 revealed the heterozygous $\mathrm{C} \rightarrow \mathrm{T}$ transition at nucleotide position 754, which resulted in substitution of an arginine codon (CGC) by a cysteine codon (TGC) at amino acid position 252 (R252C), as shown in the upper panel, whereas the normal sequence is shown in the middle panel. Verification of the paternal mutation by digestion with Nar I is shown in the lower panel. The mutation abolishes the restriction enzyme site for Nar I, which cuts the 378-bp PCR product in case of normal allele to 238-bp, 75-bp, and 65-bp bands, and in case of the mutant allele to 238-bp and 140-bp bands. $M W$, molecular weight markers $\phi$ X174/HaeIII.

genetic lesion. In addition, one allele containing a missense mutation (L336P) (family E) also revealed the presence of a putative splice site mutation $(3112-3 \mathrm{C} \rightarrow \mathrm{T})$ in combination with a missense mutation $\mathrm{R} 1225 \mathrm{H}$ in trans. Among the probands, two were homozygous for their mutation (families $\mathrm{F}$ and $\mathrm{G}$ ), whereas the remainder were compound heterozygotes (Table 1). In each case, the mutations disclosed in the patients' DNA were confirmed in their parents in the heterozygous state. Thus, EB-PA in each case was inherited in an autosomal recessive manner. The mutations discovered in this study bring the total number of mutations in ITGB4 to 33 (Table 2). In addition to pathogenetic mutations, a number of neutral sequence variations in ITGB4 were discovered in this study
(Table 3). Most of these polymorphisms were relatively rare, found in only a small number (less than five) of the normal controls screened.

Examination of DNA from 100 unrelated control individuals did not reveal either the presence of the missense mutations or a putative splicing mutation, indicating that they were not common polymorphisms. Furthermore, all missense mutations affected amino acid residues that were conserved between human, rat, and mouse ITGB4, although not all of them were conserved between different human $\beta$ integrin chains or the $\beta \nu$ integrin chain ( $\beta$ neu) in Drosophila (Fig. 4). It should be noted that these missense mutations, with the exception of $\mathrm{R} 1225 \mathrm{H}$, which resides in the fibronectin III-like domain in the intracel- 
Table 2. ITGB4 mutations reported in the literature and in this study

\begin{tabular}{|c|c|c|c|c|}
\hline Case no. & Mutations* & Consequences $\dagger$ & Affected exons & References \\
\hline \multicolumn{5}{|c|}{ Lethal } \\
\hline 1 & $1141 \mathrm{delC} / 3793+2 \mathrm{insT}$ & $\mathrm{PTC} / \mathrm{Spl}$ & $10 / 30$ & 17 \\
\hline 2 & 3434delT/4050del 8 & PTC/PTC & $28 / 32$ & 18 \\
\hline 3 & $3793+1 \mathrm{G} \rightarrow \mathrm{A} / 3793+1 \mathrm{G} \rightarrow \mathrm{A}$ & $\mathrm{Spl} / \mathrm{Spl}$ & $30 / 30$ & 10 \\
\hline 4 & 4501delTC/4501delTC & $\mathrm{PTC} / \mathrm{PTC}$ & $34 / 34$ & 11 \\
\hline 5 & 120delTG/C245G & PTC/missense & $3 / 7$ & 11 \\
\hline 6 & Q73X/ND & $\mathrm{PTC} / \mathrm{ND}$ & $4 / \mathrm{ND}$ & 11 \\
\hline 7 & C738X/4791delCA & $\mathrm{PTC} / \mathrm{PTC}$ & $18 / 36$ & 12 \\
\hline 8 & $\mathrm{C} 61 \mathrm{Y} / \mathrm{C} 61 \mathrm{Y}$ & Missense/missense & $4 / 4$ & 12 \\
\hline 9 & $658 \mathrm{delC} / \mathrm{R} 252 \mathrm{C}$ & PTC/missense & $7 / 8$ & This study (family A) \\
\hline 10 & $\mathrm{D} 131 \mathrm{Y} / \mathrm{G} 273 \mathrm{D}$ & Missense/missense & $5 / 8$ & This study (family B) \\
\hline 11 & 1874delTCTinsC/V325D & PTC/missense & $16 / 8$ & This study (family D) \\
\hline 12 & Q1767X/Q1767X & PTC/PTC & $41 / 41$ & This study (family F) \\
\hline \multicolumn{5}{|c|}{ Nonlethal } \\
\hline 13 & $\mathrm{C} 562 \mathrm{R} / \mathrm{C} 562 \mathrm{R}$ & Missense/missense & $14 / 14$ & 12 \\
\hline 14 & $\mathrm{R} 1281 \mathrm{~W} / \mathrm{R} 252 \mathrm{C}$ & Missense/missense & $31 / 8$ & 12 \\
\hline 15 & $\mathrm{R} 1281 \mathrm{~W} / \mathrm{R} 1281 \mathrm{~W}$ & Missense/missense & $31 / 31$ & 12 \\
\hline 16 & $\mathrm{~L} 156 \mathrm{P} / \mathrm{R} 554 \mathrm{X}$ & Missense/PTC & $5 / 14$ & 13 \\
\hline 17 & $3793+1 \mathrm{G} \rightarrow \mathrm{A} / \mathrm{W} 1478 \mathrm{X}$ & $\mathrm{Spl} / \mathrm{PTC}$ & $30 / 34$ & 19 \\
\hline 18 & C38R/4776delG & Missense/PTC & $3 / 36$ & 19 \\
\hline 19 & $3976-19 \mathrm{~T} \rightarrow \mathrm{A} / 3793+1 \mathrm{G} \rightarrow \mathrm{A}$ & $\mathrm{Spl} / \mathrm{Spl}$ & $32 / 30$ & 20 \\
\hline 20 & $\mathrm{ND} / \mathrm{R} 283 \mathrm{C}$ & $\mathrm{ND} /$ missense & $\mathrm{ND} / 8$ & This study (family C) \\
\hline 21 & $3112-3 \mathrm{C} \rightarrow \mathrm{T}, \mathrm{L} 336 \mathrm{P} / \mathrm{R} 1225 \mathrm{H}$ & Spl, missense/missense & $27,9 / 30$ & This study (family E) \\
\hline 22 & 4790delTC/4790delTC & $\mathrm{PTC} / \mathrm{PTC}$ & $36 / 36$ & This study (family G) \\
\hline
\end{tabular}

* Nucleotide numbers refer to the translation-initiation codon (ATG) as being $+1 /+3$ in $\beta 4$ integrin cDNA sequence (GenBank accession nos. X53587 and NM000213)

$\dagger N D$, not determined; $S p l$, putative splicing mutation.

lular segment, are found within the extracellular domain of the $\beta 4$ integrin subunit, thus possibly interfering with ligand binding and/or $\alpha 6 / \beta 4$ subunit interactions.

The mutations resulting in PTC as a result of nonsense mutations or small out-of-frame insertions or deletions predict synthesis of a truncated polypeptide and/or down-regulation of the ITGB4 mRNA levels as a result of nonsense-mediated mRNA decay $(29,30)$. Thus, no functional $\beta 4$ integrin polypeptides are synthesized, apparently resulting in EB-PA phenotype in affected individuals.

Examination of the ITGB4 mutation database reveals genotype/phenotype correlations. Identification of the 11 novel mutations in this study brings the total number of distinct $\beta 4$ integrin mutations disclosed thus far in EB-PA to 33 (Table 2 and Fig. 5). These mutations have been found in a total of 22 individuals with EB-PA, 12 of them being classified as lethal and 10 as nonlethal cases (Table 2). Examination of the mutation database in ITGB4 reveals a predominance of PTCcausing mutations in the lethal variant of EB-PA, whereas missense mutations are more prevalent in the nonlethal variant. In fact, at least 13 out of 23 alleles in 12 lethal cases harbor PTC mutations $(57 \%)$ and 7 alleles are missense mutations $(30 \%)$, whereas, in cases of nonlethal variants, at least 5 out of 19 alleles in 10 individuals are PTC mutations (26\%) and 11 alleles are missense mutations (58\%).

Missense/missense mutations associated with a lethal phenotype. Two cases with a "lethal" variant of EB-PA carried missense/missense combinations, suggesting that the position or the type of these mutations influences the phenotype. However, one of these cases (case 8 in Table 2) with homozygous $\mathrm{C} 61 \mathrm{Y} / \mathrm{C} 61 \mathrm{Y}$ mutations died during the first week of life before surgery for pyloric atresia was performed. Thus, the consequence of this mutation to clinical outcome remains unclear (12). The second case (case 10 in Table 2) is a compound heterozygote for D131Y/G273D missense mutations with entirely negative immunostaining for $\alpha 6$ and $\beta 4$ integrin epitopes with antibodies recognizing the extracellular domains of the protein. The corresponding aspartic acid-131 and glycine-273 are located in a highly conserved region (Fig. 4). Furthermore, it has been shown that several missense mutations in the corresponding region in $\beta 3$ integrin abolish ligand-binding activity $(31,32)$. Thus, this region in $\beta 4$ integrin may have ligand-binding properties similar to those of $\beta 3$ integrin. Based on these observations, the mutations D131Y and G273D are predicted to abolish ligand binding of $\beta 4$ integrin, and could at least partially explain the severe phenotype of the patient.

Missense/PTC mutations associated with a lethal phenotype. Three other lethal cases studied previously and in this work (cases 5, 9, and 11 in Table 2) are compound heterozygotes for PTC and missense mutations. In all of them, the PTC mutations may cause mRNA decay or, alternatively, result in the synthesis of truncated nonfunctional $\beta 4$ integrin polypeptides. In the absence of functional $\beta 4$ integrin polypeptide from the PTC allele, the missense mutation will determine the phenotype of each patient. One of them (case 9) harbors the mutation R252C, which has been previously characterized in a nonlethal patient (case 14) in combination with another missense mutation R1281W (12). When mutation R252C is combined with a PTC mutation, the phenotype is lethal, suggesting more severe consequences of this mutation on the function of $\beta 4$ integrin than mutation R1281W. Case 5 harbors the cysteine-to-glycine substitution at position 245 (C245G), which, 
Table 3. Nonpathogenetic nucleotide variations in the ITGB4 gene

\begin{tabular}{|c|c|c|c|c|c|}
\hline \multirow[b]{2}{*}{ Location } & \multirow[b]{2}{*}{ Nucleotide position } & \multicolumn{2}{|c|}{ Nucleotide } & \multirow{2}{*}{$\begin{array}{c}\text { Restriction } \\
\text { enzyme site* }\end{array}$} & \multirow[b]{2}{*}{ Allele frequencies } \\
\hline & & Major & Minor & & \\
\hline Exon 6 & 513 & $\mathrm{~T}(\mathrm{Phe})$ & $\mathrm{C}(\mathrm{Phe})$ & $\operatorname{Hinf} \mathrm{I}$ & $0.991 / 0.009$ \\
\hline Exon 15 & 1821 & $\mathrm{G}$ (Ser) & A (Ser) & Tsp45 I & $0.932 / 0.068$ \\
\hline Intron 19 & $2255-41$ & $\mathrm{G}$ & A & $\operatorname{Hinf} \mathrm{I}$ & $0.958 / 0.042$ \\
\hline Exon 27 & 3240 & $\mathrm{C}$ (His) & $\mathrm{T}$ (His) & Nla III & $0.983 / 0.017$ \\
\hline Exon 41 & 5567 & $\mathrm{G}(\mathrm{Arg})$ & $\mathrm{A}(\mathrm{Gln})$ & $M s p \mathrm{I}$ & $0.988 / 0.012$ \\
\hline
\end{tabular}

* These enzymes are used for confirmation of the nucleotide changes; $B f a \mathrm{I}$ in parenthesis requires the use of specific primers.

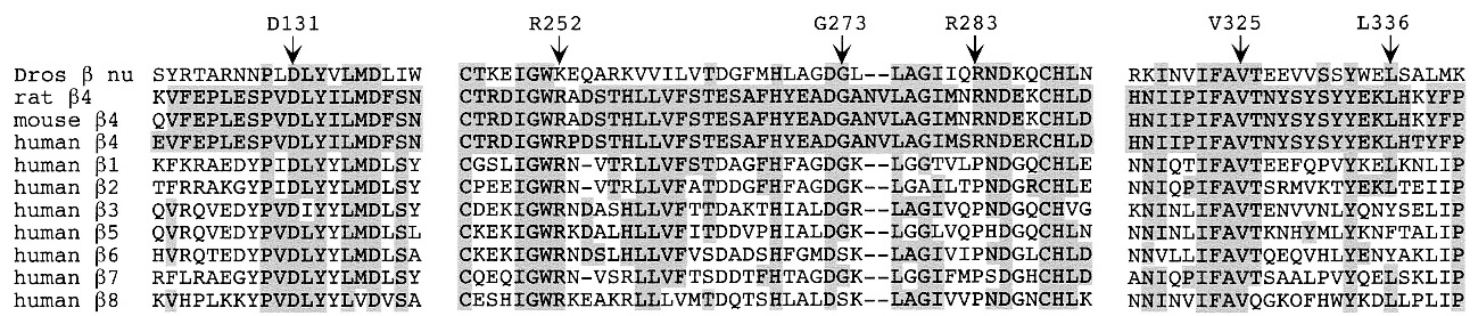

Figure 4. Comparison of human integrin $\beta 4$ polypeptide chain, spanning the regions containing the aspartic acid, arginine, glycine, arginine, valine, and leucine residues affected by mutations at positions $131,252,273,283,325$, and 336, respectively, with rat and mouse $\beta 4$ integrins and Drosophila $\beta \nu$ ( $\beta$ neu) chain, as well as with human $\beta 1-\beta 8$ integrins. The shaded regions indicate conserved amino acids. GenBank accession numbers for $\beta$ integrin genes are as follows: rat $\beta 4$, NM013180; mouse $\beta 4$, L04678; human $\beta 4$, X53587, NM000213; Drosophila $\beta \nu$, L13305; human $\beta 1$, X07979; human $\beta 2$, NM000211; human $\beta 3$, NM002210; human $\beta 5$, X53002; human $\beta 6$, NM000888; human $\beta 7$, NM000889, M62880, M68892; and human $\beta 8$, NM002214.

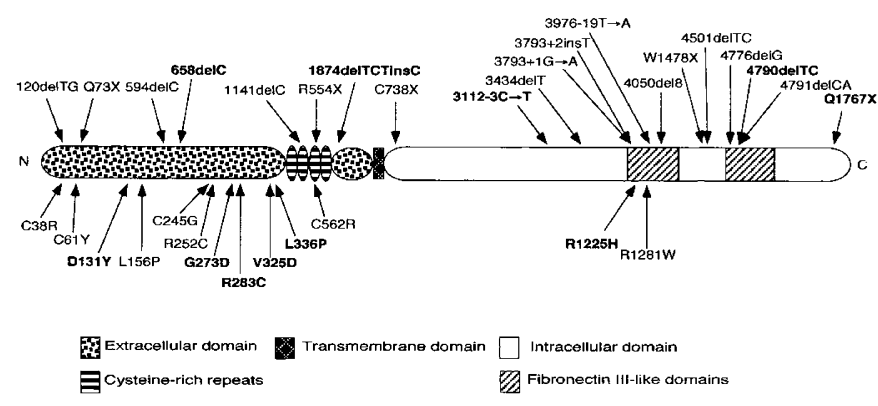

Figure 5. Schematic illustration of the $\beta 4$ integrin polypeptide with domain organization and positions of all ITGB4 mutations disclosed thus far in patients with EB-PA. The PTC and putative splice site mutations are shown above the molecule, whereas missense mutations are shown below the molecule. Novel mutations disclosed in this study are shown in bold.

along with arginine-252, is a conserved amino acid located in a putative ligand-binding region (32). When in the hemizygous state, these mutations may be especially deleterious inasmuch as creating or abolishing cysteine residues may change disulfide bonding and alter the secondary structure of the polypeptide. Such conformational changes may result in the loss of the putative ligand-binding function of the $\beta 4$ integrin. The missense mutation V325D (case 11 in Table 2) substitutes a nonpolar for an acidic residue and also occurs in a position relatively conserved between different $\beta$ integrin chains as well as between rat and human $\beta 4$ integrin (Fig. 4).

Mutations associated with a nonlethal phenotype. Among the nonlethal EB-PA cases, three of them were either compound heterozygous (cases 17 and 19) or homozygous (case 22 , family G) for putative PTC-causing mutations and three were either compound heterozygous or homozygous for mis- sense mutations (cases 13, 14, and 15) (Table 2). In two of the nonlethal cases, severe nephrosis was reported (cases 15 and 21) (see Ref. 12 and this study). One of these patients is homozygous for $\mathrm{R} 1281 \mathrm{~W}$, whereas another patient harbors two putative mutations, a splice site $(3112-3 \mathrm{C} \rightarrow \mathrm{T})$ and a missense (L336P) on one allele, and a missense mutation (R1225H) on the other allele. The mutations $\mathrm{R} 1281 \mathrm{~W}$ and $\mathrm{R} 1225 \mathrm{H}$ are located specifically within the first pair of fibronectin III-like domains in the intracellular domain. This region is involved in the $\beta 4$ integrin interaction with plectin as well as in directing its hemidesmosomal localization (33-35). It should be noted that the BMZ-specific staining of plectin was reduced in all patients, indicating that absent or mutated $\beta 4$ integrin polypeptides apparently disturb the interaction between plectin and $\beta 4$ integrin. In addition, in case 21 (family E), the putative splice site mutation affects the nucleotide at position -3 at the intron 26/exon 27 border, indicating that this mutation may cause alternatively spliced products potentially affecting exon 27 . On the other hand, leucine at position 336 is conserved between rat, mouse, and human $\beta 4$ integrin, whereas the human $\beta 3, \beta 5$, and $\beta 6$ integrin chains have different amino acids in the corresponding position (Fig. 4). Therefore, leucine may play a less critical role at this position and only minor effects of mutations at this site are predicted. These observations could explain the mild phenotype in the case 21 (family E).

Case 17 was a compound heterozygote for $3793+1 \mathrm{G} \rightarrow \mathrm{A}$ and W1478X. Although the mutation $3793+1 \mathrm{G} \rightarrow \mathrm{A}$ has been previously shown to result in at least two different frameshift splice variants affecting exon 30 , recent studies have suggested that functional splicing can be restored, at least in vitro, by changing the culture conditions of keratinocytes (20). Thus, it 
is conceivable that the mutation $3793+1 \mathrm{G} \rightarrow \mathrm{A}$ may result, under certain circumstances in vivo, in some functional $\beta 4$ integrin polypeptides, perhaps explaining the milder, nonlethal phenotype with a nonsense mutation, W1478X, in trans. Case 22 (family G), with a nonlethal clinical presentation, is homozygous for a PTC/PTC mutation, 4790delTC/4790delTC. This mutation predicts truncation of the $\beta 4$ integrin polypeptide close to the carboxyl-terminal end, potentially eliminating approximately $14 \%$ of the polypeptide (Fig. 4). The 4790delTC mutation seems not to cause total absence of mRNA through nonsense-mediated decay, inasmuch as the skin of the patient demonstrated the presence, although in reduced amounts, of $\alpha 6$ and $\beta 4$ integrin polypeptides (Fig. 2). In contrast, in case 12 (family F), the nonsense mutation Q1767X results in a stop codon on both alleles, from which we can predict deletion of 39 amino acids from the C-terminus of the $\beta 4$ integrin polypeptide. This patient, who is from a remote Aboriginal community, died at about 2.5 mo of age with a relatively mild skin condition compared with other lethal cases. This emphasizes the importance of continuing care and the possible role of other clinical manifestations on the outcome. TEM findings in case 12 (family F) revealed intracellular cleavage in the skin, a finding that is reinforced by recent studies that have shown that this region of the $\beta 4$ integrin polypeptide is critical for the interaction with $\mathrm{BP} 180(34,36)$. Because tissue for immunofluorescence was not available from this patient, the $\alpha 6$ and $\beta 4$ integrin staining patterns could not be studied.

In two patients listed in Table 2, the second mutation was not identified despite screening and complete sequencing of the ITGB4 gene. The possible promoter region mutations resulting in absent expression of ITGB4 from one allele was investigated by sequencing the ITGB4 promoter region from genomic DNA in these patients without evidence of mutations. Unfortunately, RNA was not available for reverse transcription-PCR studies in either case. Large genomic deletions were ruled out by Southern blots of the ITGB4 region, and the identification of heterozygosity for several intragenic polymorphisms further supported this finding. The second mutations in these two cases, therefore, remain undetected.

In summary, with a few exceptions, an overall theme explaining the severity of the EB-PA phenotype, lethal versus nonlethal, seems to be emerging from these studies. In general, a combination of missense mutations on both alleles may be associated with a milder skin phenotype depending upon the functional significance of the affected amino acids within the protein, whereas PTC in both alleles cause a severe phenotype. However, the phenotype of the patient is clearly influenced by the position of the missense mutation in the functional domains of the $\beta 4$ integrin protein. The missense mutations in the putative ligand-binding domain cause a severe phenotype, especially when the mutations affect conserved amino acids, whereas mutations affecting less conserved amino acids cause milder phenotypes. It should be noted that the immunofluorescence studies in this and previous works correlate relatively well with the phenotype, i.e. in nonlethal cases $\alpha 6 \beta 4$ integrin expression is positive whereas in lethal cases this protein is absent. This emphasizes the importance of immunofluorescence examination, in addition to mutation analysis, as a diagnostic procedure with prognostic implications for EB-PA patients. Precise prediction of the phenotype will require further knowledge of the interactions of ITGB4 with other structural proteins in the skin and other affected tissues. This will be accomplished, in part, by further examination of the expanding ITGB4 mutation database in patients with EB-PA and their associated phenotypes, as well as by further characterization of the functional domains of the $\alpha 6 \beta 4$ integrin molecule through experimental studies.

Acknowledgments. The authors thank the families participating in this study. Dr. J.-D. Fine, National Epidermolysis Bullosa Registry, University of North Carolina, Chapel Hill, assisted in diagnostic evaluation and provided electron microscopy and immunofluorescence service to the patients. Dr. A. Sonnenberg, the Netherlands Cancer Institute, Amsterdam, Netherlands; Dr. K. Owaribe, Nagoya University, Nagoya, Japan; and Dr. P. Marinkovich, Stanford University, Palo Alto, $\mathrm{CA}$, provided the Mab recognizing $\alpha 6$ integrin $(\mathrm{GoH} 3), \beta 4$ integrin (3E1), plectin (HD121), and type VII collagen (NP185). Dr. G. Bennett, Canberra Hospital, assisted with the electron micrographs, and D. Bhargava, St George Hospital, Sydney, and C.W. Chow, Royal Children's Hospital, Melbourne, assisted with antibody staining.

\section{REFERENCES}

1. Fine J-D, Bauer EA, McGuire J, Moshell A 1999 Epidermolysis Bullosa: Clinical, Epidemiologic, and Laboratory Advances and the Findings of the National Epidermolysis Bullosa Registry. Johns Hopkins Press, Baltimore, pp 300-325

2. Fine J-D, Eady RAJ, Bauer EA, Briggaman RA, Bruckner-Tuderman L, Christiano A, Heagerty A, Hintner H, Jonkman MF, McGrath J, McGuire J, Moshell A, Shimizu H, Tadini G, Uitto J 2000 Revised classification system for inherited epidermolysis bullosa: report of the Second International Consensus Meeting on Diagnosis and Classification of Epidermolysis Bullosa. J Am Acad Dermatol 42:1051-1066

3. Christiano AM, Uitto J 1996 Molecular complexity of the cutaneous basement membrane zone: revelations from the paradigms of epidermolysis bullosa. Exp Dermatol 5:1-11

4. Pulkkinen L, Uitto J 1999 Mutation analysis and molecular genetics of epidermolysis bullosa. Matrix Biol 18:29-42

5. Pulkkinen L, Uitto J 1998 Hemidesmosomal variants of epidermolysis bullosa: mutations in the $\alpha 6 \beta 4$ integrin and the $180-\mathrm{kD}$ bullous pemphigoid antigen/type XVII collagen genes. Exp Dermatol 7:46-64

6. Hashimoto I, Schnyder UW, Anton-Lamprecht I 1976 Epidermolysis bullosa hereditaria with junctional blistering in an adult. Dermatologica 152:72-86

7. Hintner H, Wolff K 1982 Generalized atrophic benign epidermolysis bullosa. Arch Dermatol 118:375-384

8. Uitto J, Pulkkinen L, Smith FJD, McLean WHI 1996 Plectin and human genetic disorders of the skin and muscle: the paradigm of epidermolysis bullosa with muscular dystrophy. Exp Dermatol 5:237-246

9. Pulkkinen L, Kimonis VE, Xu Y, Spanou EN, McLean WHI, Uitto J 1997 Homozygous $\alpha 6$ integrin mutation in junctional epidermolysis bullosa with congenital duodenal atresia. Hum Mol Genet 6:669-674

10. Pulkkinen L, Kurtz K, Xu Y, Bruckner-Tuderman L, Uitto J 1997 Genomic organization of the integrin $\beta 4$ gene (ITGB4): a homozygous splice-site mutation in a patient with junctional epidermolysis bullosa associated with pyloric atresia. Lab Invest 76:823-833

11. Pulkkinen L, Kim DU, Uitto J 1998 Epidermolysis bullosa with pyloric atresia: novel mutations in the $\beta 4$ integrin gene (ITGB4). Am J Pathol 152:157-166

12. Pulkkinen L, Rouan F, Bruckner-Tuderman L, Wallerstein R, Garzon M, Brown T, Smith L, Carter W, Uitto J 1998 Novel ITGB4 mutations in lethal and nonlethal variants of epidermolysis bullosa with pyloric atresia: missense versus nonsense. Am J Hum Genet 63:1376-1387

13. Pulkkinen L, Bruckner-Tuderman L, August C, Uitto J 1998 Compound heterozygosity for missense (L156P) and nonsense (R554X) mutations in the $\beta 4$ integrin gene (ITGB4) underlies mild, nonlethal phenotype of epidermolysis bullosa with pyloric atresia. Am J Pathol 152:935-941

14. Gil SG, Brown TA, Ryan MC, Carter WG 1994 Junctional epidermolysis bullosa: defects in expression of epiligrin/nicein/kalinin and integrin $\beta 4$ that inhibit hemidesmosome formation. J Invest Dermatol 103:31S-38S

15. Brown TA, Gil SG, Sybert VP, Lestringant GG, Tadini G, Caputo R, Carter WG 1996 Defective integrin $\alpha 6 \beta 4$ expression in the skin of patients with junctional epidermolysis bullosa and pyloric atresia. J Invest Dermatol 107:384-391 
16. Niessen CM, Raaij-Helmer LMH, Hulsman EHM, Neut R, Jonkman MF, Sonnenberg A 1996 Deficiency of the integrin $\beta 4$ subunit in junctional epidermolysis bullosa with pyloric atresia: consequences for hemidesmosome formation and adhesion properties. J Cell Sci 109:1695-1706

17. Vidal F, Aberdam D, Miquel C, Christiano AM, Pulkkinen L, Uitto J, Ortonne J-P, Meneguzzi G 1995 Integrin $\beta 4$ mutations associated with junctional epidermolysis bullosa with pyloric atresia. Nat Genet 10:229-234

18. Takizawa Y, Shimizu H, Nishikawa T, Hatta N, Pulkkinen L, Uitto J 1997 Novel ITGB4 mutations in a patient with junctional epidermolysis bullosa-pyloric atresia syndrome and altered basement membrane zone immunofluorescence for the $\alpha 6 \beta 4$ integrin. J Invest Dermatol 108:943-946

19. Mellerio JE, Pulkkinen L, McMillan JR, Lake BD, Horn HM, Tidman MJ, Harper JI, McGrath JA, Uitto J, Eady RAJ 1998 Pyloric atresia-junctional epidermolysis bullosa syndrome: mutations in the integrin $\beta 4$ gene (ITGB4) in two unrelated patients with mild disease. Br J Dermatol 139:862-871

20. Chavanas S, Gache Y, Vailly J, Kanitakis J, Pulkkinen L, Uitto J, Ortonne J-P, Meneguzzi G 1999 Splicing modulation of integrin $\beta 4$ pre-mRNA carrying a branch point mutation underlies epidermolysis bullosa with pyloric atresia undergoing spontaneous amelioration with aging. Hum Mol Genet 8:2097-2105

21. Ruzzi L, Gagnoux-Palacios L, Pinola M, Belli S, Meneguzzi G, D'Alessio M, Zambruno G 1997 A homozygous mutation in the integrin $\alpha 6$ gene in junctional epidermolysis bullosa with pyloric atresia. J Clin Invest 99:2826-2831

22. Gache Y, Romero-Graillet C, Spadafora A, Lépinard C, Descamps P, Bardon CB Ortonne JP, Meneguzzi G 1998 A novel homozygous mutation affecting integrin $\alpha 6$ in a case of junctional epidermolysis bullosa with pyloric atresia detected in utero by ultrasound examination. J Invest Dermatol 111: 914-916

23. Sambrook J, Fritsch EF, Maniatis T 1989 Molecular Cloning: A Laboratory Manual Cold Spring Harbor Laboratory Press, Plainview, NY, pp 9.16-9.19

24. Ganguly A, Rock MJ, Prockop DJ 1993 Conformation-sensitive gel electrophoresis for rapid detection of single-base differences in double-stranded PCR products and DNA fragments: evidence for solvent-induced bends in DNA heteroduplexes. Proc Natl Acad Sci U S A 90:10325-10329

25. Körkkö J, Annunen S, Pihlajamaa T, Prockop DJ, Ala-Kokko L 1998 Conformation sensitive gel electrophoresis for simple and accurate detection of mutations: comparison with denaturing gradient gel electrophoresis and nucleotide sequencing. Proc Natl Acad Sci U S A 95:1681-1685
26. Groot WG, Postuma R, Hunter AGW 1978 Familial pyloric atresia associated with epidermolysis bullosa. J Pediatr 92:429-431

27. Lestringant GG, Akel SR, Qayed KI 1992 The pyloric atresia-junctional epidermolysis bullosa syndrome; report of a case and review of the literature. Arch Dermatol 128:1083-1086

28. Maman E, Maor E, Kachko L, Carmi R 1998 Epidermolysis bullosa, pyloric atresia, aplasia cutis congenita: histopathological delineation of an autosomal recessive disease. Am J Med Genet 78:127-133

29. Frischmeyer PA, Dietz HC 1999 Nonsense-mediated mRNA decay in health and diseases. Hum Mol Genet 8:1893-1900

30. Hentze MW, Kulozik AE 1999 A perfect message: RNA surveillance and nonsensemediated decay. Cell 96:307-310

31. Tozer EC, Liddington RC, Sutcliffe MJ, Smeeton AH, Loftus JC 1996 Ligand binding to integrin $\alpha_{\mathrm{IIb} \beta 3}$ is dependent on a MIDAS-like domain in the $\beta 3$ subunit. J Biol Chem 271:21978-21984

32. Baker EK, Tozer EC, Pfaff M, Shattil SJ, Loftus JC, Ginsberg MH 1997 A genetic analysis of integrin function: Glanzmann thrombasthenia in vitro. Proc Natl Acad Sci U S A 94:1973-1978

33. Borradori L, Koch PJ, Niessen CM, Erkeland S, Leusden MR, Sonnenberg A 1997 The localization of bullous pemphigoid antigen 180 (BP180) in hemidesmosomes is mediated by its cytoplasmic domain and seems to be regulated by the $\beta 4$ integrin subunit. J Cell Biol 136:1333-1347

34. Schaapveld RQJ, Borradori L, Geerts D, Leusden MR, Kuikman I, Nievers MG, Niessen CM, Steenbergen RDM, Snijders PJF, Sonnenberg A 1998 Hemidesmosome formation is initiated by the $\beta 4$ integrin subunit, requires complex formation of $\beta 4$ and HD1/plectin, and involves a direct interaction between $\beta 4$ and the bullous pemphigoid antigen 180. J Cell Biol 142:271284

35. Nievers MG, Schaapveld RQJ, Sonnenberg A 1999 Biology and function of hemidesmosomes. Matrix Biol 18:5-17

36. Aho S, Uitto J 1998 Direct interaction between the intracellular domains of bullous pemphigoid antigen 2 (BP180) and $\beta 4$ integrin, hemidesmosomal components of basal keratinocytes. Biochem Biophys Res Commun 243:694-699 